\title{
Mechanical Behavior Analysis of A Test Coil for MICE Coupling Solenoid during Quench
}

\author{
H. Pan, L. Wang, X. L. Guo, H. Wu, and M. A. Green, Member, IEEE
}

\begin{abstract}
The coupling magnet for the Muon Ionization Cooling Experiment has a self-inductance of $592 \mathrm{H}$ and the magnet stored energy of $13 \mathrm{MJ}$ at a full current of $210 \mathrm{~A}$ for the worst operation case of the MICE channel. The high level of stored energy in the magnet can cause high peak temperature during a quench and induce considerable impact of stresses. One test coil was built in order to validate the design method and to practice the stress and strain situation to occur in the coupling coil. In this study, the analysis on stress redistribution during a quench with sub-divided winding was performed. The stress variation may bring about failure of impregnating material such as epoxy resin, which is the curse of a new normal zone arising. Spring models for impregnating epoxy and fiber-glass cloth in the coil were used to evaluate the mechanical disturbance by impregnated materials failure. This paper presents the detailed dynamic stress and stability analysis to assess the stress distribution during the quench process and to check whether the transient loads are acceptable for the magnet.
\end{abstract}

Index Terms-Dynamic stress, MICE superconducting magnet, Quench, Stability.

\section{INTRODUCTION}

$\mathrm{T}$ he superconducting coupling solenoid to be applied in the Muon Ionization Cooling Experiment (MICE) is made of copper matrix $\mathrm{Nb}-\mathrm{Ti}$ conductors with inner radius of $750 \mathrm{~mm}$, length of $285 \mathrm{~mm}$ and thickness of $110.4 \mathrm{~mm}$ at room temperature. The magnetic field up to $2.6 \mathrm{~T}$ at the magnet centerline is to keep the muons within the MICE RF cavities. Its self inductance is around $592 \mathrm{H}$ and its magnet stored energy is about $13 \mathrm{MJ}$ at a full current of $210 \mathrm{~A}$ for the worst operation case of the MICE channel [1].

A high temperature rise in the region where a quench originally started will occur because of the high stored energy and the high current densities. The stresses inside it during a quench are relatively high due to the large size of the coupling magnet and thermal expansion coefficient difference among different materials. A test coil was designed and constructed in order to validate the magnet design method and to practice the

Manuscript received 20 October 2009. This work was supported by Funds of cryogenics and superconductivity technology innovation project under "985-2 Plan" of Harbin Institute of Technology, China and by the Office of Science, US Department of Energy under DOE contract DE-AC02-05CH11231.

H. Pan, H. Wu, X. L. Guo are with the Institute of Cryogenics and Superconductivity Technology, Harbin Institute of Technology, Harbin 150001, China (phone: 86-451-86412066, e-mail: panheng@hit.edu.cn).

L. Wang was with the Institute of Cryogenics and Superconductivity Technology, HIT, Harbin 150001, China. She is now with Shanghai Institute of Applied Physics, Shanghai 201204, China (e-mail: wangli icst@hit.edu.cn).

M. A. Green is with Lawrence Berkeley National Laboratory, Berkeley, CA, 94720 USA (e-mail: magreen@lbl.gov). stress and strain situation to be encountered in the coupling coil. The test coil has the same layers of 96 and $1.5 \mathrm{~m}$ inner diameter as the coupling coil but with one-quarter length of the coupling coil [2].

The test coil adopted the copper matrix $\mathrm{Nb}$-Ti conductors exactly same as those used for the MICE coupling magnet. The maximum exciting current of the test coil is designed to be $400 \mathrm{~A}$, at which the strain condition to happen in the coil are to be greater than that would be encountered in the coupling coil. This paper presents the detailed analyses on stresses in the test coil assembly during a quench by the finite element method, in order to study the structure safety and stability of the magnet in a quench. The time-dependent map of the variation of stress distribution within the coil is computed using a commercial code. The instability due to strain energy releasing during a quench based on a spring model is discussed as well.

\section{MODEL OF THE TEST COIL}

The test coil assembly consists of the coil package, the banding and the coil mandrel. The banding for the coil reinforcement is made of stainless steel wire with diameter of $1.2 \mathrm{~mm}$. The mandrel includes one bobbin, two end plates and one cover plate, which made of 6061-T6 aluminum alloy. The insulation between the coil package and the mandrel is thin G-10 sheets; the interlayer insulation is epoxy and fiber-glass cloth, and the insulation between turns is epoxy resin. The self-inductance is around $50.8 \mathrm{H}$. The peak induction in the test coil is $6.78 \mathrm{~T}$ and its magnet stored energy is about 4.064 $\mathrm{MJ}$ at the maximum current of $400 \mathrm{~A}$. The cross section of the test coil assembly is shown in Fig. 1. Table I lists the basic dimensions of the test coil.

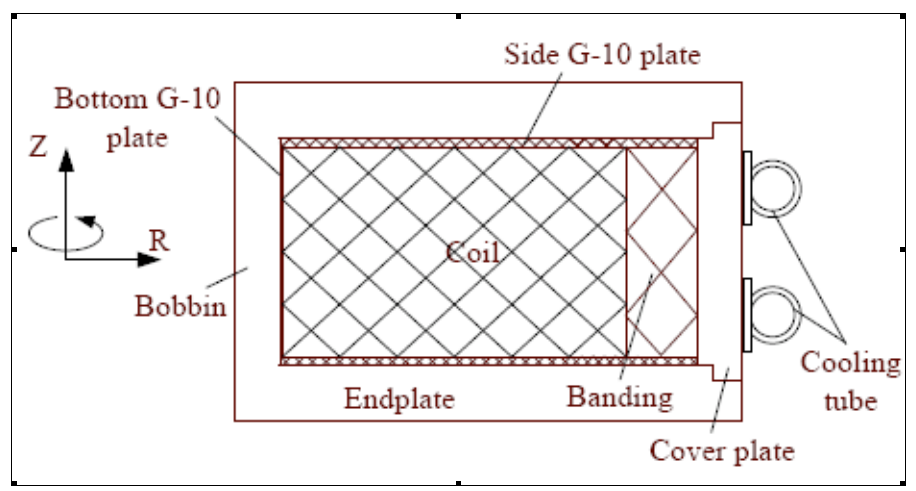

Fig. 1. The cross-section of the test coil 
TABLE I The Basic Dimensions of the Test Coil Assembly

\begin{tabular}{cc}
\hline \hline Parameters & Thickness $(\mathbf{m m})$ \\
\hline Bobbin & 13 \\
End plate & 18 \\
Cover plate & 15 \\
Banding & 27 \\
Bottom G-10 plate & 1 \\
Side G-10 plate & 3 \\
\hline \hline
\end{tabular}

A 2-D axial symmetric finite element model was developed to calculate the dynamic stresses within the coil assembly during a quench. It involved two parts: one was to calculate the time-dependent temperature and the decaying current, the other was to calculate the static and dynamic stress distribution within the coil. In the quench computational model, the quench is assumed to start in the middle of the innermost layer of the coil. The temperature and the current were coupled and sequentially solved. The transient current was loaded to calculate joule heat, and then the temperature from the joule heat calculation was loaded to calculate the transient current. The calculation model doesn't include diodes and damp resistors, and is not sub-divided in order to simplify the calculation. The hot spot temperature of the quench calculation model is about $10 \mathrm{~K}$ higher than the result from the sub-divided quench model [2]. The analysis results in this paper may represent the worst case for the coil during a quench in terms of hot spot temperature and are more conservative.

The stress model calculates the stress distribution before a quench, firstly. And then, the time-dependent temperature and the magnetic load were used as input for calculating the dynamic stress. The stress model adopted the element birth and death technique to simulate the winding process. The multi-load method is used to simulate the cooling, charging and quench process sequentially. In this model, the coil was simulated as a single block of conductors with uniform and linear material properties, and all parts were glued together.

\section{COMPUTATION RESULTS AND DISCUSSION}

\section{A. The Results of a Quench Model}

Disturbance energy was injected to the coil to generate a normal zone. With the normal zone growing, the current decayed and the hot spot temperature rose. There was inductive current in the mandrel and the banding, which generated heat. If the temperature of the mandrel or the banding is higher than the critical temperature, the quench-back effect will happen [3].

Variations of the hot spot temperature and the magnetic field with the quench time in the test coil are shown in Fig. 2. After about $5 \mathrm{~s}$, the magnetic field decreases to below $1 \%$ of the initial magnetic field. The peak coil hot spot temperature is $135 \mathrm{~K}$, which is about $10 \mathrm{~K}$ higher than the result of 4 sub-divided coil charged to the same current. The difference of the results with and without subdivision is not obvious for the stress calculation [4].

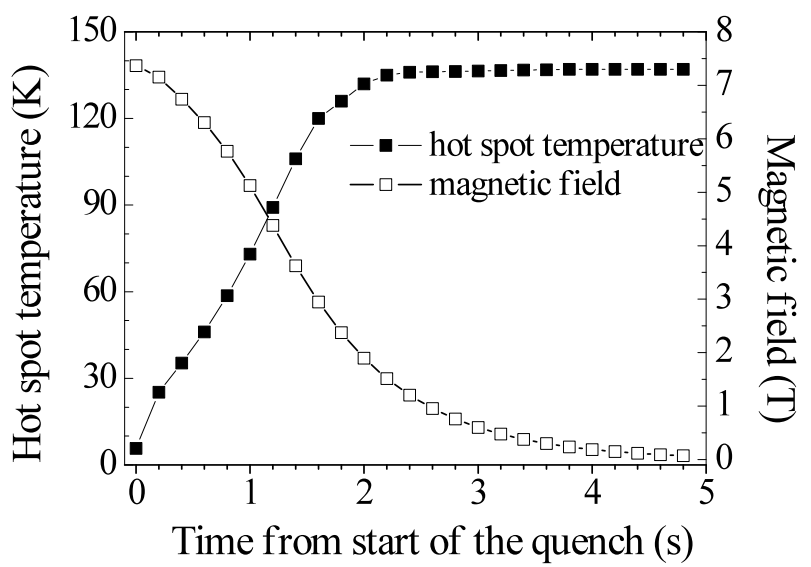

Fig. 2. Magnetic field decay and hot spot temperature rise through the coil after a quench

\section{B. The Dynamic Stress Distribution}

In this paper, we assume that a quench starts after the coil is charged to the full current. Therefore, the static stress distribution exists before the quench starts. The followed stresses during the quench are the sum of the static stress and the stress change because of variation of the magnetic field and the temperature. The following study is concentrated on the dynamic stress analyses in terms of the mechanical safety and the new normal zone generation. For the coil with slip planes, the radial stress and the shear stress are more responsible for the stability.

To follow the transient behavior of the test coil, two loads are applied in the model: (1) the Lorentz body force decreasing from their maximum value at $I=400 \mathrm{~A}, B=7.4 \mathrm{~T}$ to zero when $I=0 \mathrm{~A}$; (2) the temperature rising with respect to time in the quenched coil. The integrated expansion is assumed between $4 \mathrm{~K}$ to $200 \mathrm{~K}$, thus the results calculated are that in a bad case.

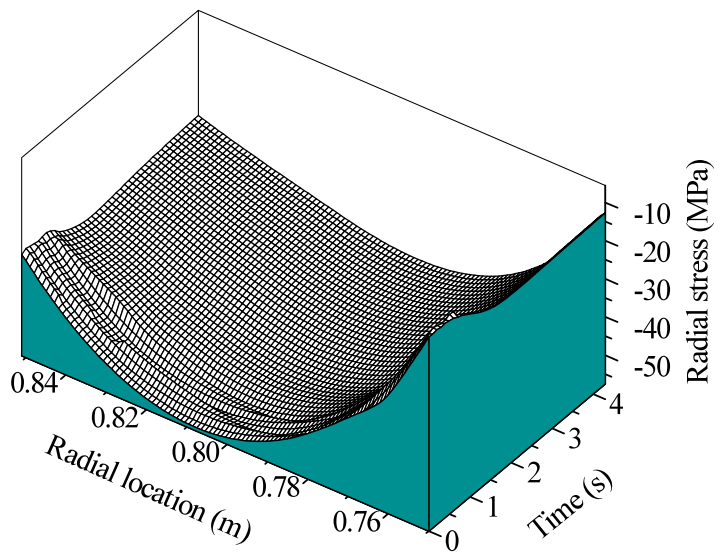

Fig. 3. Radial stress in the mid-plane of the coil during the quench.

Fig. 3 shows the radial stress distribution along the mid-plane $(z=0)$ of the coil during the quench. The radial pressure releases is characterized by the decreasing of the peak radial pressure because the Lorentz force reduces during the 
quench. The radial pressure in the interface between the coil and the mandrel increases a little during the quench, but still less than the value after cooling down. It may be interpreted that the thermal expansion of the normal zone makes the inner layers to move outward, which replaces the radial Lorentz body force during the quench.

The peak radial stress, which moves to the inner layers of the coil during the quench, decreases with time. That means a part of the strain energy of the central layers of the coil has been released. The maximum change rate of the radial displacement is about $0.046 \%$ happened in the outermost layers of the coil. If the radial strain is less than $0.065 \%$, the coil will avoid quench or training [5]. Therefore, the radial stress during the quench will not cause a new normal zone.

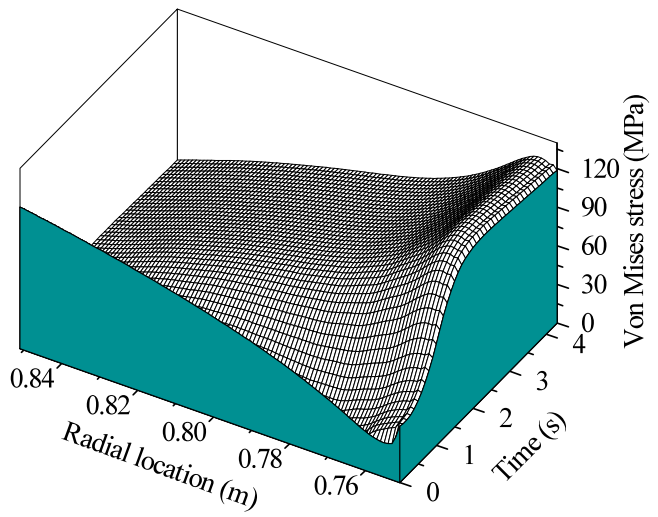

Fig. 4. Von Mises stress in the mid-plane of the coil during the quench.

Fig. 4 shows the Von Mises stress distribution along the mid-plane $(z=0)$ of the coil during the quench. When the exciting current goes to the maximum current of $400 \mathrm{~A}$, the peak Von Mises stress in the coil package is about $110 \mathrm{MPa}$, which appears in the outermost layer of the coil caused by the radial Lorentz force before the quench started. While quench ends, the peak stress moves to the innermost layer of the coil, and is about $120 \mathrm{MPa}$. The peak stress during the quench is less than the allowable stress of the conductor. As shown in Fig. 4, the stress level in the inner layers of the coil increases rapidly within $2 \mathrm{~s}$. The equivalent strain rate is about $0.024 \%$ $\mathrm{s}^{-1}$. The rapid change of stress will cause the micro-crack in the brittle material [6]. So the variation of the Von Mises stress may cause a rise of new points of initiation of the normal zone.

Fig. 5 shows the peak shear stress in the coil during the quench. The peak shear stress happens in the innermost corner of the coil. Its change with quench time is the same as the peak temperature in the coil. Compared with other stress distribution, the peak shear stress is mainly affected by the temperature rising within the coil rather than the magnetic field.

The maximum value of the peak shear stress during the quench is about $80 \mathrm{MPa}$ by the finite element method. Actually, the impregnating material can not withstand such a high stress, and it will crack under the stress higher than the tensile or shear strength. The Stycast epoxy was employed in the test coil, which is brittle at $4.2 \mathrm{~K}$. It has very low fracture stress, even lower than its shear strength of $17 \mathrm{MPa}$ [7]. When the major principal stress or the maximum shear stress in the shear stress concentrate region is higher than the fracture stress, the epoxy resin will crack and the strain energy of this region will dissipate as heat.

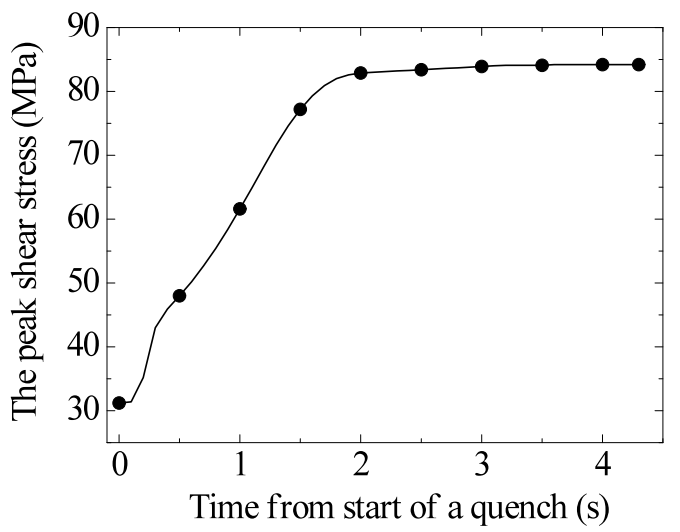

Fig. 5. The peak shear stress in the innermost corner of the coil during the quench.

When the tensile strain is more than $2 \%$ or shear strain is more than $0.2 \%$, the epoxy resin will crack [8]. When the shear stress is higher than $40 \mathrm{MPa}$, the shear strain of epoxy resin will exceed $0.2 \%$, which means a new normal zone may generate at the innermost corner. The mechanism of stress to induce a new normal zone is discussed in the following section.

Additionally, the peak hoop stress is about $-120 \mathrm{MPa}$ at the end of the quench. The peak tensile hoop stress is about 50 MPa before the quench started. The peak hoop stress during the quench never exceeded the allowable stress of the conductor.

In conclusion, the increase of the temperature and the simultaneous reduction of the electromagnetic forces will not damage the coil assembly. But the stress variation may induce a number of now points of the normal zone in the inner layers of the coil, especially the shear stress. Therefore, it is concluded that the shear stress is the critical factor on the instability and cause more disturbance during a quench.

\section{MECHANICAL DISTURBANCE CALCULATION}

\section{A. The Spring Model Description}

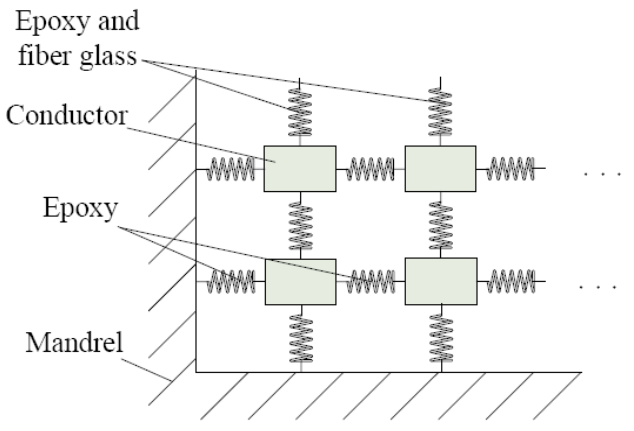

Fig. 6. Spring model to calculate mechanical disturbance. 
In the impregnated magnet, the source of cracking of the impregnating material is mainly the thermal stress or Lorentz force. The cracking is more affected by the thermal stress according to the above analyses. With the totally elastic deformation assumption, the impregnating material can be treated as spring under small deformation [9]. Fig. 6 shows the schematic illustration of the spring model to calculate the mechanical stability. In the spring model, we assume that the spring failure corresponds with the epoxy cracking, and half of the elastic energy stored in springs will released as heat.

The interlayer and interturn insulations of the test coil are fiber-glass cloth immersed in epoxy and only epoxy, respectively. Corresponding to the spring model as shown in Fig. 7, the interlayer insulation is a kind of spring with elastic coefficient $k^{I}$, and the interturn insulation is the spring with elastic coefficient $k^{I I}$. It has four constraints from the surrounding springs, thus there are force equilibrium under external loads for each conductor. The springs can deflect, so the direction of a spring is the major principal stress orientation of the impregnated material. The force situation under arbitrary conditions for each conductor is.

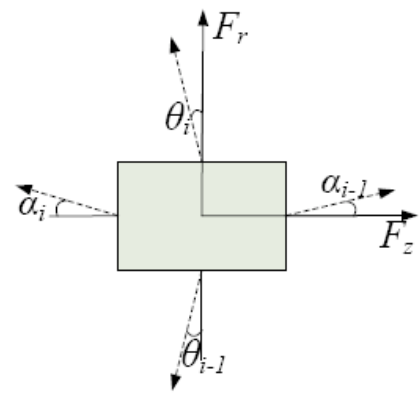

Fig. 7. Force situation of conductor $i$

So, the equilibrium equations of conductor $i$ are given as follows:

$k_{i}^{I} \varepsilon_{i}^{I} \cos \theta_{i}+k_{i-1}^{I} \varepsilon_{i-1}^{I} \cos \theta_{i-1}+k_{i}^{I I} \varepsilon_{i}^{I I} \sin \alpha_{i}$

$+k_{i-1}^{I I} \varepsilon_{i-1}^{I I} \sin \alpha_{i-1}+F_{r}=0$

$k_{i}^{I} \varepsilon_{i}^{I} \cos \alpha_{i}+k_{i-1}^{I} \varepsilon_{i-1}^{I} \cos \alpha_{i-1}+k_{i}^{I I} \varepsilon_{i}^{I I} \sin \theta_{i}$

$+k_{i-1}^{I I} \varepsilon_{i-1}^{I I} \sin \theta_{i-1}+F_{z}=0$

where $\varepsilon^{I}$ is the deformation of interlayer spring; $\varepsilon^{I I}$ is the deformation of interturn spring; $F_{r}$ and $F_{z}$ are body force in radial and axial directions.

\section{B. Results and Discussion}

We focused on the innermost corner in the coil because the stress concentrates in this region. The number of conductor involved is about 150, which in the axial direction is 6 and in the radial direction is 25 . Therefore, there are totally 150 equilibrium equations. The load conditions are: (1) when the coil is not charged, the body force in each conductor is zero, and there is only displacement constraint; (2) when the coil is charged or in a quench process, the body force is the magnetic load and the simultaneous displacement in each conductor.
From the calculation, the maximum deformation of springs located between the first turn of the innermost layer and the endplate. The destruction mode is shear failure. Table II lists the results of this spring.

The maximum shear strain decreases remarkably when the coil is charged to 400 A because the hoop stress at that point increases. So, the epoxy cracking will take place during cooling down, and cause training during charging. When the coil quenched, the rising shear stress will induce a new normal zone.

TABLE II The Results of the Maximum Deformation Spring

\begin{tabular}{cc}
\hline \hline Process & Maximum shear strain \\
\hline Cooling down & $1.2 \%$ \\
Charging to 400 A & $0.475 \%$ \\
After quench & $1.9 \%$ \\
\hline \hline
\end{tabular}

\section{CONCLUSION}

The dynamic stress for the test coil of the MICE coupling magnet during a quench is studied by using a commercial code. The temperature rising of the normal zone affects the stress distribution more remarkable than the simultaneous reduction of the electromagnetic force, especially the peak shear stress.

The instability of the test coil due to the failure of epoxy is calculated by a spring model. The shear stress in the innermost corner of the coil is principally responsible for premature quenches and training effect.

\section{REFERENCES}

[1] Institute of cryogenics and superconductivity Technology, "Muon Ionization Cooling Experiment Coupling Solenoid Magnet Engineering Design Report," (Unpublished) Harbin Institute of Technology, China, Dec. 2008.

[2] L. Wang, H. Pan, F. Y. Xu, et al. "Design and Construction of Test Coils for MICE Coupling Solenoid Magnet," IEEE Trans. on Appl. Super., vol 19, No. 3, pp 1340-1343, June, 2009.

[3] M. A. Green, "Quench Back in Thin Superconducting Solenoid Magnets". Cryogenics 24, p 3-10, 1984.

[4] N. V. Krivolutskaya, O. A. Kleshnina, and P. N. Lebedev, "Stresses in Superconducting Solenoid Winding during its Quench," IEEE Trans. on Magn., vol 30, No. 4, pp 2547-2549, July, 1994.

[5] V. R. Karasik, O. A. Kleshnina, A. A. Konjukhov, V. A. Malginov, and P. N. Lebedev, "High current density superconducting magnet with 2.58 m Bore," Proc. Adv. in Cryo. Engi., New York, 1992, p 40-46.

[6] Z .T. Guan, Z. T. Zhang, and J. S. Jiao. Physical Properties of Inorganic Material. Beijing, Tsinghua University Press, 1992, ch.2.

[7] S. Yadav, and J. Hoffman, "Epoxies for Cryogenic Applications," Fermi Lab Note, TD-99-021, 1999.

[8] E. S. Bobrov, J. E. C. Williams, and Y. Iwasa, "Experimental and theoretical investigation of mechanical disturbances coils. 2 . Shear-stress-induced epoxy fracture as the principal source of premature quenches and training-theoretical analysis," Cryogenics, vol 25, pp 307-316, June 1985.

[9] S. Ohira, S. Nishijima, "Effects of impregnating material failure on stability of superconducting magnet analyzed by wire dynamics simulation," IEEE Trans. on Appl. Super., vol 10, No. 1, pp 665-668, March, 2000. 